\title{
HOMOLOGY OF BRANCHED CYCLIC COVERS OF KNOTS
}

\author{
STANLEY OCKEN \\ (Communicated by Frederick R. Cohen)
}

\begin{abstract}
This paper presents a new formula for the first integral homology group of the branched cyclic $p$-fold cover $\Sigma_{p}$ of a knot $K$ in the 3-sphere. Given a diagram of $K$ with $k$ crossings, let $A(t)$ be the $(k-1) \times(k-1)$ Alexander matrix of the diagram. Let $C=A(1)^{-1} A(0)$, and let $I$ be the identity matrix. Then $(C-I)^{p}-C^{p}$ is a presentation matrix for $H_{1}\left(\Sigma_{p}\right)$.
\end{abstract}

This paper presents an efficient new algorithm for computing the homology of branched cyclic covers of a knot $K$ in $S^{3}$. The classical procedure ([F], [S]) for such computation employs as input data a Seifert matrix of $K$, obtained by calculating linking numbers of homology cycles on a spanning surface of the knot. The new approach described here relies instead on the Alexander matrix, which can be obtained directly from a knot diagram and is much easier to compute. Our results show how to read the required information from the knot diagram in an essentially canonical way and avoid any reference to the Seifert surface of $K$.

Given a diagram of $K$ with $k$ crossings, the Alexander matrix of $K$ corresponding to the diagram is a $k \times k$ matrix whose rows each contain entries $1-t, t,-1$ in columns determined by the fundamental group relation at the crossings of the diagram [F]. Let $A(t)$ be the $(k-1) \times(k-1)$ matrix obtained by crossing out one row and one column of the Alexander matrix. Then $A(t)$ is a presentation matrix for the first integral homology of the infinite cyclic cover of $S^{3}-K$, viewed as a $Z\left[t, t^{-1}\right]$ module, where the action of $t$ on homology is induced by the covering translation generated by a knot meridian. Note that the matrix $A(t)$ corresponding to a particular diagram of $K$ is well defined up to permutation of rows and columns, multiplication of a row by -1 , and the choice of the row and column omitted from the Alexander matrix. Theorem 2 below shows a particular choice of conventions which reduces to a minimum the computation in the following theorem.

Theorem 1. Let $A(t)$ be as above, and let $C$ be the integer matrix $A(1)^{-1} A(0)$.

Received by the editors April 11, 1988 and, in revised form, March 11, 1989.

1980 Mathematics Subject Classification (1985 Revision). Primary 57M12.

Key words and phrases. Branched cyclic coverings. 
Then for any integer $p \geq 2$,

$$
(C-I)^{p}-C^{p}
$$

is a presentation matrix for the first integral homology group of the p-fold branched cyclic cover of $K$.

The matrix $A(t)$ described above depends on the method used to number the segments of the diagram. The computationally optimal method is as follows. Pick a starting point on the diagram, and label the segments of the knot consecutively in the order that a point traverses the knot. Define $\varepsilon(n)$ to be -1 if the crossing following segment $n$ is right-handed; otherwise let $\varepsilon(n)$ be +1 . In either case, let $p(n)$ be the number of the oversegment at this crossing. Here is a picture:

$$
\varepsilon=-1: \frac{n}{p} \uparrow_{p(n)} \stackrel{s(n)}{\longrightarrow} \quad \varepsilon=+1: \stackrel{s(n)}{\longleftarrow} \uparrow_{p(n)} \underline{n}
$$

The successor to knot segment $n$ is of course $s(n)=n(\bmod k)+1$. Finally, let $\tau(n)$ be $n($ resp. $s(n))$ if $\varepsilon(n)$ is -1 (resp. +1$)$.

Given these conventions, let $A(t)$ be the matrix obtained by crossing out row $k$ and column $k$ of the Alexander matrix described above. The next result shows that the segment numbering convention just described leads to an extremely simple computation of the homology of branched cyclic covers.

Theorem 2. With the above choice of segment numbers,

(a) $A(1)^{-1}$ is the $(k-1) \times(k-1)$ matrix

$$
\left(\begin{array}{llllllll}
1 & 1 & 1 & 1 & 1 & \cdots & 1 & 1 \\
0 & 1 & 1 & 1 & 1 & \cdots & 1 & 1 \\
0 & 0 & 1 & 1 & 1 & \cdots & 1 & 1 \\
& \cdot & \cdot & \cdot & . & \cdots & . & \\
& \cdot & \cdot & \cdot & . & \cdots & . & \\
0 & 0 & 0 & 0 & 0 & \cdots & 1 & 1 \\
0 & 0 & 0 & 0 & 0 & \cdots & 0 & 1
\end{array}\right) .
$$

(b) For $1 \leq n \leq k-1$, row $n$ of $A(0)$, the Alexander matrix evaluated at $t=0$,

(i) is entirely zero if $p(n)=\tau(n)$;

(ii) contains $\varepsilon(n)$ in column $p(n),-\varepsilon(n)$ in column $\tau(n)$, and zero elsewhere if $p(n) \neq \tau(n)$.

Thus the matrix $C$ of Theorem 1 can be computed from a quick look at the knot diagram.

Proof of Theorem 1. Given a diagram of $K$ with $k$ crossings, orient the knot and number the segments of the diagram from 1 to $k$ in any order. Also, 
assign numbers 1 to $k$ to the crossings in the diagram. Let $\alpha_{1}, \alpha_{2}, \ldots, \alpha_{k} \in$ $\pi_{1}\left(S^{3}-K\right)$ be the meridian generators which loop around the $k$ segments of the diagram. At crossing $n$, there is a fundamental group relation

$$
\alpha_{a(n)} \alpha_{b(n)}=\alpha_{c(n)} \alpha_{a(n)}
$$

where $\alpha_{a(n)}$ is the generator corresponding to the oversegment at that crossing and $\alpha_{b(n)}$ and $\alpha_{c(n)}$ are the undersegments; here $a, b$, and $c$ are permutations of $1,2, \ldots, k$. The Alexander matrix of the diagram is the $k \times k$ matrix in which the nonzero entries in row $n$ are $1-t, t$, and -1 in columns $a(n)$, $b(n)$, and $c(n)$ respectively.

Let $A(t)$ be the matrix obtained by crossing out a row and a column of the Alexander matrix. In order to compute the homology of the $p$-fold branched cyclic cover $\Sigma_{p}$ of $S^{3}-K$, observe that the entries of $A(t)$ are linear in $t$, and so we can write

$$
A(t)=A(0)+t(A(1)-A(0)) .
$$

As remarked in [G], ${ }^{1}$ a presentation matrix $R$ for $H_{1}\left(\Sigma_{p}\right)$ is obtained by replacing each entry $a+b t$ of $A(t)$ by the $p \times p$ matrix $a I+b T$, where $I$ is the identity matrix and $T$ is the matrix

$$
\left(\begin{array}{lllllll}
0 & 1 & 0 & 0 & \cdots & 0 & 0 \\
0 & 0 & 1 & 0 & \cdots & 0 & 0 \\
0 & 0 & 0 & 1 & \cdots & 0 & 0 \\
& . & . & . & \cdots & . & \\
& . & . & . & \cdots & . & \\
0 & 0 & 0 & 0 & \cdots & 0 & 1 \\
1 & 0 & 0 & 0 & \cdots & 0 & 0
\end{array}\right) .
$$

It follows easily, after rearrangement of rows and columns, that $R$ consists of $p^{2}$ blocks, each of size $k-1$ by $k-1$, as follows:

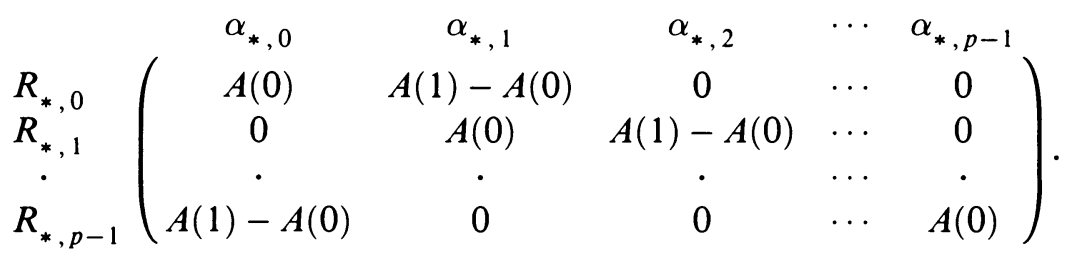

Here $A(t)$ is the $(k-1) \times(k-1)$ matrix obtained by crossing out the column (resp. row) corresponding to $\alpha_{k}$ (resp. $R_{k}$ ) from the Alexander matrix of $K$. We may rewrite $R$ in a more convenient form by setting $M=A(1)-A(0)$, i.e. $A(0)=A(1)-M=A(1)(I-C)$, where $I$ is the identity matrix and $C=I-A(1)^{-1} A(0)$. If we multiply each row of blocks in $R$ by the unimodular

\footnotetext{
${ }^{1}$ The author thanks the referee for suggesting this reference.
} 
matrix $A(1)^{-1}, R$ takes the form

$$
\left(\begin{array}{cccccc}
I-C & C & 0 & 0 & \cdots & 0 \\
0 & I-C & C & 0 & \cdots & 0 \\
0 & 0 & I-C & C & \cdots & 0 \\
\dot{C} & \dot{0} & 0 & 0 & \cdots & \cdot \\
\hline & 0 & I-C
\end{array}\right) .
$$

It follows as in [S] that this presentation matrix can be reduced via row and column operations to the $(k-1) \times(k-1)$ matrix

$$
(C-I)^{p}-C^{p}
$$

as desired.

Proof of Theorem 2. We now show how to compute efficiently. Given an oriented knot diagram with $k$ crossings, number the segments of the diagram sequentially from 1 to $k$ while traversing the knot once in the direction of the given orientation. For each segment $n$ of the knot, let $\alpha_{n}$ be the corresponding generator of $\pi_{1}\left(S^{3}-K\right)$, which loops under segment $n$ in the direction to the right of the orientation of the segment. Now number each knot crossing with the segment number of the incoming undersegment at that crossing. Define the orientation $\varepsilon(n)$ of crossing $n$ to be -1 if the crossing looks like this:

$$
-\uparrow \rightarrow
$$

and 1 otherwise. Finally, define $p(n)$ to be the segment number of the oversegment at crossing $n$.

The relations among the generators implied by crossing $n$ are as follows:

$$
\begin{array}{cc}
\text { Case } \varepsilon(n)=-1 & \text { Case } \varepsilon(n)=1 \\
\stackrel{n}{\longrightarrow} \stackrel{s(n)}{\longrightarrow} & \stackrel{s(n)}{\longleftarrow} \uparrow_{p(n)}^{n} \\
\alpha_{n} \alpha_{p(n)}=\alpha_{p(n)} \alpha_{s(n)} & \alpha_{p(n)} \alpha_{n}=\alpha_{s(n)} \alpha_{p(n)}
\end{array}
$$

In the infinite cyclic cover, the corresponding relations are

$$
\begin{array}{ll}
\alpha_{n}+(t-1) \alpha_{p(n)}-t \alpha_{s(n)}=0 & (\text { if } \varepsilon(n)=-1), \text { or } \\
(1-t) \alpha_{p(n)}+t \alpha_{n}-\alpha_{s(n)}=0 & (\text { if } \varepsilon(n)=1) .
\end{array}
$$

Let $A(t)$ be the reduced matrix obtained by crossing out row $k$ and column $k$ of the Alexander matrix for $K$ obtained from the above generators and relations.

In order to describe $A(1)$ and $A(0)$, we define $h(n)$ to be equal to $n$ if $\varepsilon(n)=-1$, and $s(n)$ if $\varepsilon(n)=1$. It is easy to see that the matrix $A(0)$ can be described as follows:

(a) Row $i$ is 0 if $h(i)=p(i)$. 
(b) Otherwise, row $i$ contains $\varepsilon(i)$ in column $p(i)$ (if $p(i) \leq k),-\varepsilon(i)$ in column $h(i)$ (if $h(i) \leq k$ ), and 0 elsewhere.

In addition, the relations above both reduce to $\alpha_{n}-\alpha_{n+1}=0$ for $t=1$; hence $A(1)$ is the $(k-1) \times(k-1)$ matrix with diagonal elements equal to 1 , elements immediately above the diagonal equal to -1 , and all other elements equal to 0 . Hence $A(1)^{-1}$ is the $(k-1) \times(k-1)$ matrix with entries equal to 1 on or above the main diagonal, and 0 below.

Note that the product $C=A(1)^{-1} A(0)$ can be calculated with fewer than $2 k$ additions, rather than the $(k-1)^{3}$ additions and multiplications which would normally be required for multiplying two matrices of size $(k-1) \times(k-1)$.

\section{REFERENCES}

[F] R. H. Fox, A quick trip through knot theory, Topology of 3-Manifolds and Related Topics (Proc. Georgia Inst., 1961), Prentice-Hall, 1962, pp. 120-167.

[G] C. McA. Gordon, Some aspects of classical knot theory, Knot Theory (J. C. Hausmann, ed.), Lecture Notes in Math., vol. 685, Springer-Verlag, New York, 1978.

[S] H. Seifert, Die Verschlingungsinvarianten der zyklischen Knotenüberlagerungen, Abh. Math. Sem. Univ. Hamburg 11 (1935), 84-101.

Department of Mathematics, The City College, City University of New York, New YORK, NEW YORK 10031 